\title{
Tentative identification of urea and formamide in ISO-SWS infrared spectra of interstellar ices
}

\author{
S. Raunier ${ }^{1}$, T. Chiavassa ${ }^{1}$, F. Duvernay ${ }^{1}$, F. Borget ${ }^{1}$, J. P. Aycard ${ }^{1}$, E. Dartois ${ }^{2}$, and L. d'Hendecourt ${ }^{2}$ \\ 1 Physique des Interactions Ioniques et Moléculaires, Équipe Spectrométries et Dynamique Moléculaire, UMR 6633, \\ Université de Provence, Centre de Saint Jérôme, Boîte 252, 13397 Marseille Cedex 20, France \\ 2 Institut d'Astrophysique Spatiale - CNRS, Bât 121, Université Paris-Sud, 91405 Orsay Cedex, France
}

Received 12 September 2003 / Accepted 4 November 2003

\begin{abstract}
Laboratory experiments involving vacuum ultraviolet (VUV) irradiation of solid isocyanic acid (HNCO) at $10 \mathrm{~K}$, followed by infrared spectroscopy (FTIR), are used to interpret the complex spectra associated with Interstellar Medium (ISM) dust grains, particularly the spectra associated with the icy phase observed toward dense molecular clouds. The comparison of the infrared spectra of the photolysis products with spectra recorded from the protostellar source NGC 7538 IRS9 shows that the "unexplained" $1700 \mathrm{~cm}^{-1}$ feature can be attributed to the contribution of several species $\mathrm{H}_{2} \mathrm{CO}$ (formaldehyde), $\mathrm{HCONH}_{2}$ (formamide) and $\mathrm{H}_{2} \mathrm{NCONH}_{2}$ (urea) mixed with $\mathrm{H}_{2} \mathrm{O}$ as the main contributor. Urea, formaldehyde and $\mathrm{NH}_{4}^{+} \mathrm{OCN}^{-}$(ammonium cyanate) may also contribute to a band at $1470 \mathrm{~cm}^{-1}$, widely observed in many protostellar infrared sources and which remains up to now poorly explained in numerous ISO-SWS spectra. Isocyanic acid could be a precursor of formamide and urea in interstellar ices.
\end{abstract}

Key words. stars: individual: NGC 7538 IRS9 - methods: laboratory - infrared: ISM

\section{Introduction}

Cold dust grains (10-50 K) into dense interstellar clouds are mainly composed of $\mathrm{H}_{2} \mathrm{O}, \mathrm{CO}, \mathrm{CO}_{2}, \mathrm{CH}_{3} \mathrm{OH}, \mathrm{NH}_{3}$ and some trace constituents surrounding a silicate core (Greenberg 2002). These ice mixtures can be readily identified using laboratory simulations involving "classical" matrix isolation methods (d'Hendecourt \& Dartois 2001). In the interstellar medium, under the UV irradiation field originating from hot stars, these ices are subject to photochemical processing and can evolve to form new and more complex products. Recent observations obtained from the Infrared Space Observatory (ISO) spectrometer SWS toward protostellar sources such as NGC 7538 IRS9 (Whittet et al. 1996) and W33A (Gibb et al. 2000) show the existence of features that remain poorly explained. Keane et al. (2001) claim that the absorption band observed in the $1700 \mathrm{~cm}^{-1}$ region is too broad and structured to be entirely due to $\mathrm{H}_{2} \mathrm{O}$ ice. They suggest the presence of several complex organic species containing $\mathrm{C}=\mathrm{O}$ groups. In these observations, a "XCN" band at $4.62 \mu \mathrm{m}\left(2165 \mathrm{~cm}^{-1}\right)$, assigned to $\mathrm{OCN}^{-}$ (Soifer et al. 1979; Winnewisser \& Kramer 1999; Bernstein et al. 2000; Novozamsky et al. 2001) is often detected. We assume here that the 2165 and $1700 \mathrm{~cm}^{-1}$ features are related and can have $\mathrm{HNCO}$ as precursor. $\mathrm{HNCO}$ is the simplest molecular specie in the interstellar medium (ISM) containing

Send offprint requests to: T. Chiavassa, e-mail: thierry@piimsdm.univ-mrs.fr all four most abundant elements. It has been identified in the gas phase in the ISM and in comets (Winnewisser \& Kramer 1999) but never in the solid phase on interstellar grain mantles. Nevertheless HNCO can be a transient solid state species, produced and rapidly consumed by the formation of subsequent species. HNCO can be formed in the cold dust grains by vacuum ultraviolet (VUV) photolysis of $\mathrm{CO} / \mathrm{NH}_{3}$ mixtures (d'Hendecourt et al. 1986; Grim et al. 1989) and may play a specific role in the formation of $\mathrm{NH}_{4}^{+} \mathrm{OCN}^{-}$from a purely acid-base reaction occurring at $10 \mathrm{~K}$ between $\mathrm{HNCO}$ and $\mathrm{NH}_{3}$ (Novozamsky et al. 2001; Raunier et al. 2003).

We report here the VUV irradiation of HNCO monitored by means of in-situ infrared spectroscopy as a source of formaldehyde, formamide and urea, allowing the explanation of the feature observed at $1700 \mathrm{~cm}^{-1}$ in the spectrum of NGC 7538 IRS9 protostellar ice. We show that formamide and urea, which can be at the origin of some prebiotic molecules such as pyrimidine (Robertson \& Miller 1995), are directly detected in dust grains in the ISM.

\section{Experimental setup}

The apparatus used in these experiments is an evacuated sample chamber $\left(10^{-7}\right.$ mbar $)$ containing a rotating gold-plated mirror kept at $10 \mathrm{~K}$, coupled to a Fourier Transform infrared spectrometer (Nicolet Magna 750) equipped with a liquid $\mathrm{N}_{2}$ cooled detector, a germanium-coated $\mathrm{KBr}$ beamsplitter and a globar 
source. HNCO, synthesized from the thermal decomposition of cyanuric acid (Aldrich Chemical Co., $98 \%$ ) at $650^{\circ} \mathrm{C}$, under a primary vacuum (Raunier et al. 2003) is deposited at a rate of $2 \times 10^{-2} \mathrm{~mol} \mathrm{~min}^{-1}$ onto a gold mirror, then irradiated using a microwave discharge hydrogen flow lamp (Opthos instruments), mounted directly onto the sample chamber to simulate the interstellar radiation field. The beam of the lamp, transmitted through a $\mathrm{MgF}_{2}$ window onto the substrate, is in the range 6 to $10 \mathrm{eV}$ and its spectrum is dominated by two bands centered at 120 and $160 \mathrm{~nm}$ (Hagen et al. 1979; Jenniskens et al. 1993; Cottin et al. 2003). The sample chemical evolution at $10 \mathrm{~K}$ is monitored by measuring its IR spectrum in reflection mode between 4000 and $600 \mathrm{~cm}^{-1}$ at various irradiation times. For each spectrum one hundred interferograms were accumulated and the resolution was set to $1 \mathrm{~cm}^{-1}$.

To verify the nature of the products formed during the HNCO photolysis, we successively deposited on the substrate the following species.

$\mathrm{NH}_{4}^{+} \mathrm{OCN}^{-}$was produced by thermal reaction between ammonia (Air Liquide N36, $\mathrm{H}_{2} \mathrm{O}<200$ ppmv) and $\mathrm{HNCO}$. $\mathrm{HNCO}$ and $\mathrm{NH}_{3}$ (in a ratio 1/10) were deposited at a rate of $2 \times 10^{-2} \mathrm{~mol} \mathrm{~min}^{-1}$ from two separate inlets onto the mirror held at $10 \mathrm{~K}$, at a constant pressure of $10^{-7}$ mbar. The concentration was estimated by standard manometric techniques. Pure $\mathrm{NH}_{4}^{+} \mathrm{OCN}^{-}$is obtained after sublimation of excess $\mathrm{NH}_{3}$ and residual $\mathrm{HNCO}$ at 120 and $145 \mathrm{~K}$ respectively.

Formamide $\left(\mathrm{HCONH}_{2}\right.$, Prolabo) was deposited at $10 \mathrm{~K}$ after purification by vacuum distillation.

Formaldehyde $\left(\mathrm{H}_{2} \mathrm{CO}\right)$ was prepared from paraformaldehyde (Aldrich) at $65{ }^{\circ} \mathrm{C}$ in a stainless steel U-tube then carried in argon to the mirror cooled at $50 \mathrm{~K}$ to get a solid. Because of its low vapour pressure at ambient temperature, urea $\left(\mathrm{H}_{2} \mathrm{NCONH}_{2}\right.$, Aldrich $\left.99 \%\right)$ at $10^{-7}$ mbar is heated at $50{ }^{\circ} \mathrm{C}$ then carried in argon to the mirror cooled to $50 \mathrm{~K}$.

\section{Results and discussion}

Figure 1a shows the unirradiated $\mathrm{HNCO}$ infrared spectrum at $10 \mathrm{~K}$ in the $3600-1000 \mathrm{~cm}^{-1}$ range. Three HNCO fundamental vibrational modes are observed and the frequencies are displayed in Table 1 with their assignments (Lowenthal et al. 2002; Raunier et al. 2003). The HNCO spectrum is characterized by an intense vibrational band at $2252 \mathrm{~cm}^{-1}$ and a broad feature split in two bands at $3365-3200 \mathrm{~cm}^{-1}$, which are respectively related to $v$ as $\mathrm{NCO}$ and $v \mathrm{NH}$ stretching modes. Figures $1 \mathrm{~b}$ and $1 \mathrm{c}$ show the IR spectra of the sample after 3.5 and $8.5 \mathrm{~h}$ of VUV photolysis; the positions and identifications of the IR features of each new product are listed in Table 1.

In the $2600-2100 \mathrm{~cm}^{-1}$ region, we observe that both $\mathrm{CO}$ and $\mathrm{CO}_{2}$ absorption bands at 2138 and $2343 \mathrm{~cm}^{-1}$ increase continuously during irradiation. Besides these two bands, we observe the growth of a symmetrical band at $2165 \mathrm{~cm}^{-1}$, with a width $(F W M H)$ of $25 \mathrm{~cm}^{-1}$, in good agreement with the expected frequency for $\mathrm{OCN}^{-}$(Hudson et al. 2001).

Another broad band, located near $2800 \mathrm{~cm}^{-1}$, can be ascribed to $\mathrm{NH}_{4}^{+}$, based on the spectral data of this species (Raunier et al. 2003). These observations show the presence

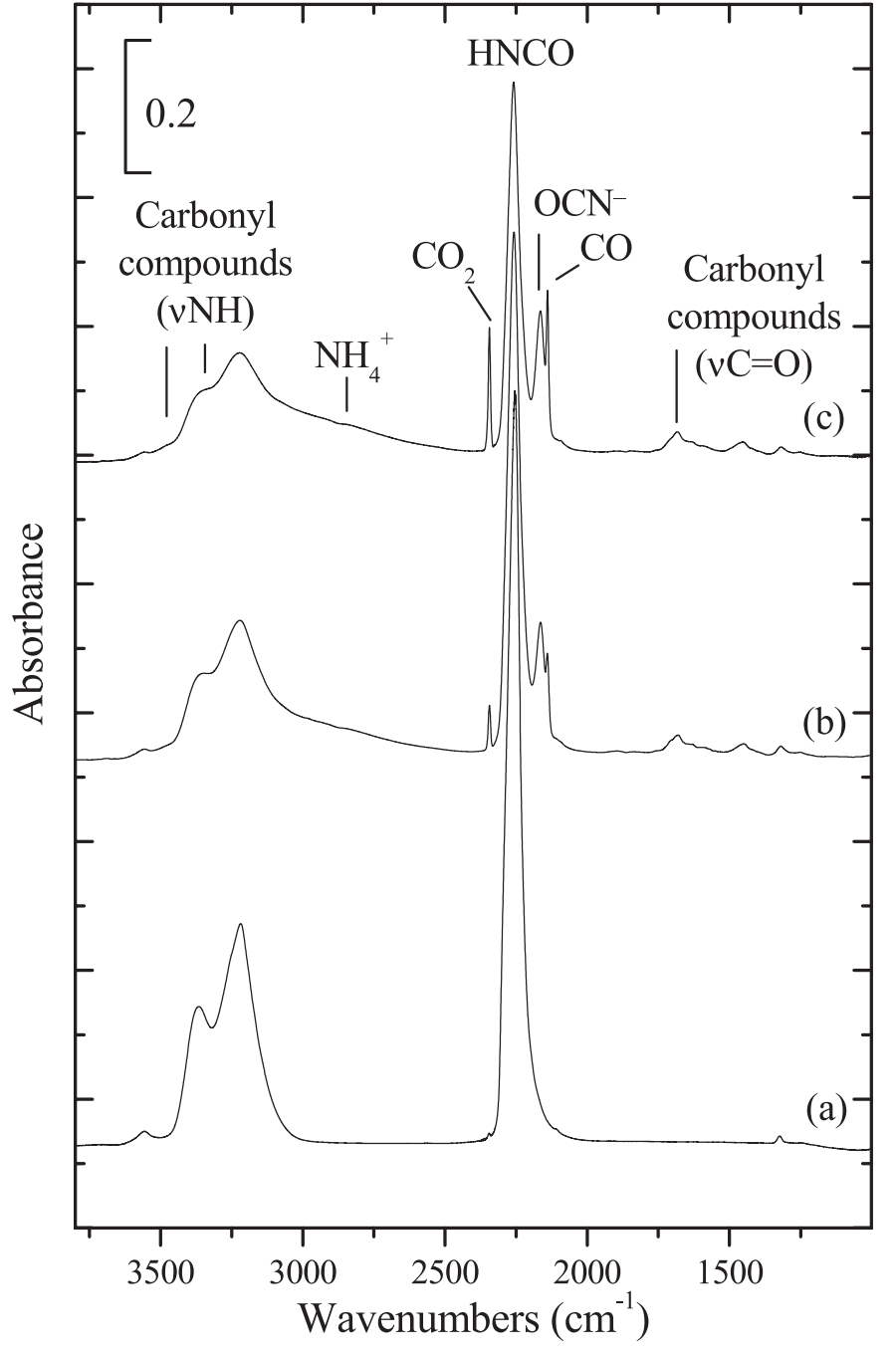

Fig. 1. VUV irradiation of solid $\mathrm{HNCO}$ at $10 \mathrm{~K}$ a) $0 \mathrm{~h}$, b) $3.5 \mathrm{~h}$, c) $8.5 \mathrm{~h}$.

of $\mathrm{NH}_{4}^{+}$and its counterion $\mathrm{OCN}^{-}$. Clear evidence of the presence of $\mathrm{NH}_{4}^{+} \mathrm{OCN}^{-}$in the mixture is obtained when the sample is warmed above $150 \mathrm{~K}$ to sublimate the residual HNCO. Under these conditions, we can observe the vibrational bands related to this species by comparison with the $\mathrm{NH}_{4}^{+} \mathrm{OCN}^{-}$spectrum obtained from the thermal reaction at $10 \mathrm{~K}$ between ammonia and $\mathrm{HNCO}$ (Fig. 2). However, during irradiation, no trace of $\mathrm{NH}_{3}$, necessary to produce $\mathrm{NH}_{4}^{+} \mathrm{OCN}^{-}$, is detected. This result is not surprising because $\mathrm{NH}_{3}$ reacts spontaneously with $\mathrm{HNCO}$ at $10 \mathrm{~K}$ and leads to $\mathrm{NH}_{4}^{+} \mathrm{OCN}^{-}$(Raunier et al. 2003). In addition, $\mathrm{NH}_{3}$ is strongly sensitive to irradiation in the VUV region and can be easily photodissociated into NH and H (Jacox \& Milligan 1964).

In the $1900-1100 \mathrm{~cm}^{-1}$ range, a broad and structured band grows at $1700 \mathrm{~cm}^{-1}$ indicating the formation of several carbonyl compounds. On the spectrum relative to the primary photoproducts recorded between 3.5 and $8.5 \mathrm{~h}$ of irradiation (Fig. 3a), we identify formaldehyde $\left(\mathrm{H}_{2} \mathrm{CO}\right)$, formamide $\left(\mathrm{HCONH}_{2}\right)$ and urea $\left(\mathrm{H}_{2} \mathrm{NCONH}_{2}\right)$ as deduced from comparison with spectra of the pure sample recorded at $10 \mathrm{~K}$. Formaldehyde is characterized by bands at 1722 
Table 1. Fundamental infrared band positions $\left(\mathrm{cm}^{-1}\right)$ with their assignments of the species produced during the VUV irradiation of solid HNCO.

\begin{tabular}{|c|c|c|c|c|c|c|c|c|}
\hline Vibration & HNCO & $\mathrm{CO}$ & $\mathrm{CO}_{2}$ & $\mathrm{OCN}^{-}$ & $\mathrm{NH}_{4}^{+}$ & $\mathrm{H}_{2} \mathrm{CO}$ & $\mathrm{HCONH}_{2}$ & $\mathrm{H}_{2} \mathrm{NCONH}_{2}$ \\
\hline $\mathrm{N}-\mathrm{H}$ stretch & $3365-3220$ & & & & 2800 & & 3316 & 3450 \\
\hline $\mathrm{N}=\mathrm{C}=\mathrm{O}_{\text {asym. }}$ stretch & 2252 & & & 2165 & & & & \\
\hline $\mathrm{N}-\mathrm{H}_{\text {sym. }}$ bend & & & & & & & & 1675 \\
\hline $\mathrm{N}-\mathrm{H}_{\text {asym. }}$ bend & & & & & & & & 1630 \\
\hline $\mathrm{C}=\mathrm{O}$ stretch & & 2138 & 2343 & & & 1722 & 1700 & 1590 \\
\hline $\mathrm{C}-\mathrm{H}_{\text {sym. }}$ bend & & & & & & 1494 & & \\
\hline $\mathrm{C}-\mathrm{N}_{\text {asym. }}$ stretch & & & & & & & & 1460 \\
\hline $\mathrm{N}-\mathrm{H}_{\mathrm{sym}}$. bend & & & & & 1470 & & 1386 & \\
\hline $\mathrm{N}=\mathrm{C}=\mathrm{O}_{\text {sym. }}$ stretch & $1322-1252$ & & & & & & & \\
\hline
\end{tabular}

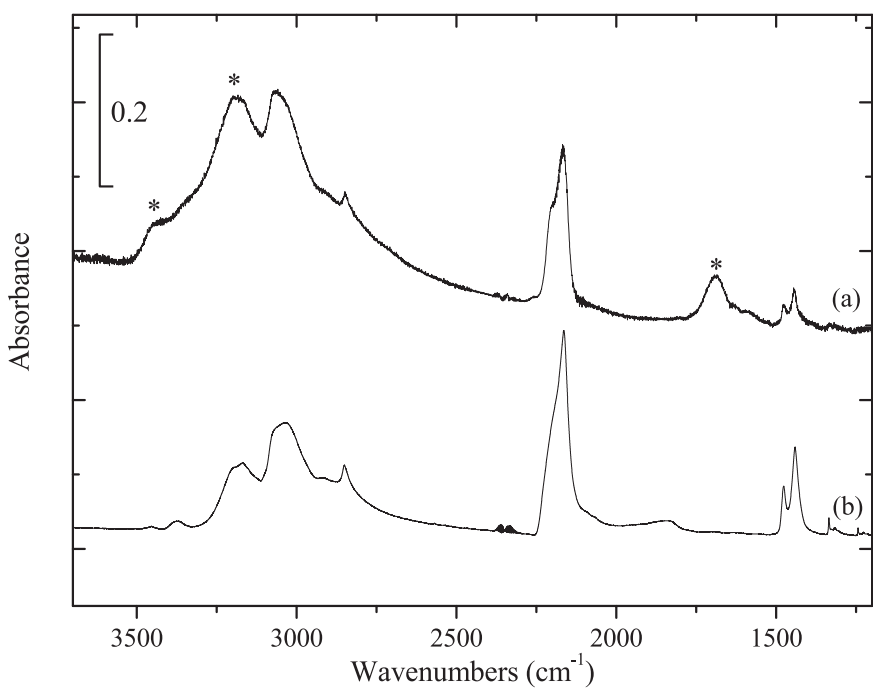

Fig. 2. Comparison of the spectra of a) the sample at $200 \mathrm{~K}$ obtained from $8.5 \mathrm{~h}$ HNCO irradiation at $10 \mathrm{~K}$ (The * show the presence in the residue of carbonyl compounds, formamide and urea) with b) $\mathrm{NH}_{4}^{+} \mathrm{OCN}^{-}$at $200 \mathrm{~K}$ obtained from a co-deposition of $\mathrm{HNCO} / \mathrm{NH}_{3}$. This spectrum is different to that recorded at $10 \mathrm{~K}$.

and $1494 \mathrm{~cm}^{-1}$, whereas formamide and urea are characterized by the band sets, $1700-1386 \mathrm{~cm}^{-1}$ and $1675-1630-1590$ $1460 \mathrm{~cm}^{-1}$. The last irradiation time $(8.5 \mathrm{~h})$ corresponds to a $50 \%$ disappearance of $\mathrm{HNCO}$ allowing one to obtain a maximum of absorption for the vibrational bands relative to the carbonyl compound products. A simple addition of the spectra corresponding to cyanate ammonium, formamide, urea (Fig. 3b) and formaldehyde (Fig. 3c) gives an excellent match with the spectrum of the irradiated sample (Fig. 3a). This confirms the formation of these carbonyl compounds during the photolysis process. It is more difficult to obtain a good fit in the $v \mathrm{NH}$ region because the HNCO band remains present. However, we note that new and weak features near $3300 \mathrm{~cm}^{-1}$ appear around 3450 and $3300 \mathrm{~cm}^{-1}$ (Fig. 1c) which are very close to the expected frequencies for solid urea (Rousseau et al. 1998) and formamide (Bukowska 1979). Our results also show a good agreement for the broad band located near $1470 \mathrm{~cm}^{-1}$ between the spectrum of the primary photoproduct irradiated (Fig. 3a) and the spectrum resulting from the addition of formaldehyde, urea and ammonium cyanate spectra (Fig. 3b).

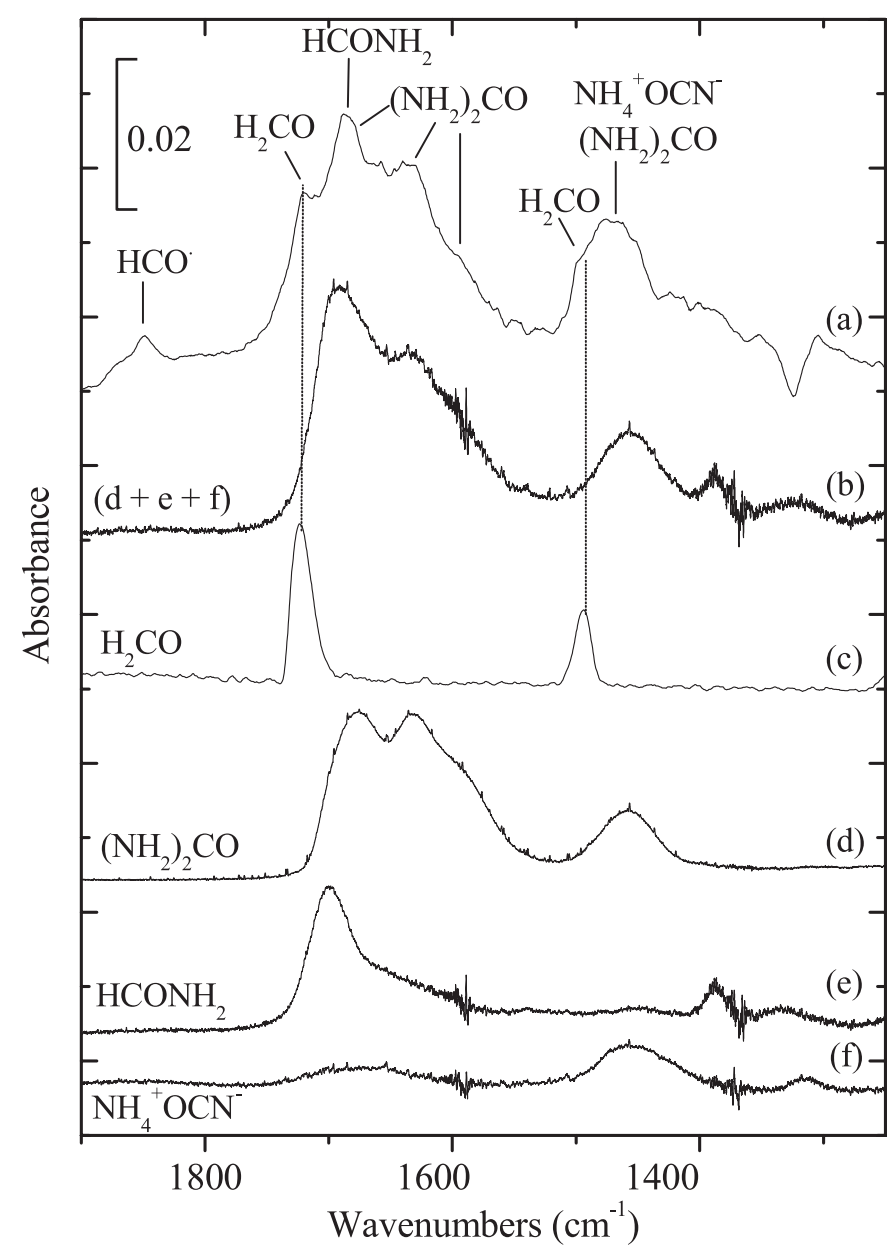

Fig. 3. Comparison between the spectra at $10 \mathrm{~K}$ of a) VUV primary photoproducts irradiated and $\mathbf{b}$ ) sum of spectra corresponding to $\mathbf{c}$ ) pure solid formaldehyde, d) pure solid urea, e) pure solid formamide and f) pure solid ammonium cyanate. The spectrum a) results from the irradiation of primary photoproducts induced from the VUV solid $\mathrm{HNCO}$ at $10 \mathrm{~K}$ and is relative to the difference spectrum recorded between the $3.5 \mathrm{~h}$ (Fig. 1b) and $8.5 \mathrm{~h}$ (Fig. 1c) irradiation times.

The spectrum of the primary irradiated photoproducts (Fig. 3a) can also be used to characterize the species existing in the interstellar ice. Molecular ice towards the young stellar object NGC 7538 IRS9 contain $\mathrm{H}_{2} \mathrm{O}$ and $\mathrm{NH}_{3}$, where $\mathrm{NH}_{3} / \mathrm{H}_{2} \mathrm{O}$ ratio is about $10 \%$. It follows the formation of ammonia hydrate $\left(\mathrm{NH}_{3} \cdot \mathrm{H}_{2} \mathrm{O}\right)$ which has an absorption band in 


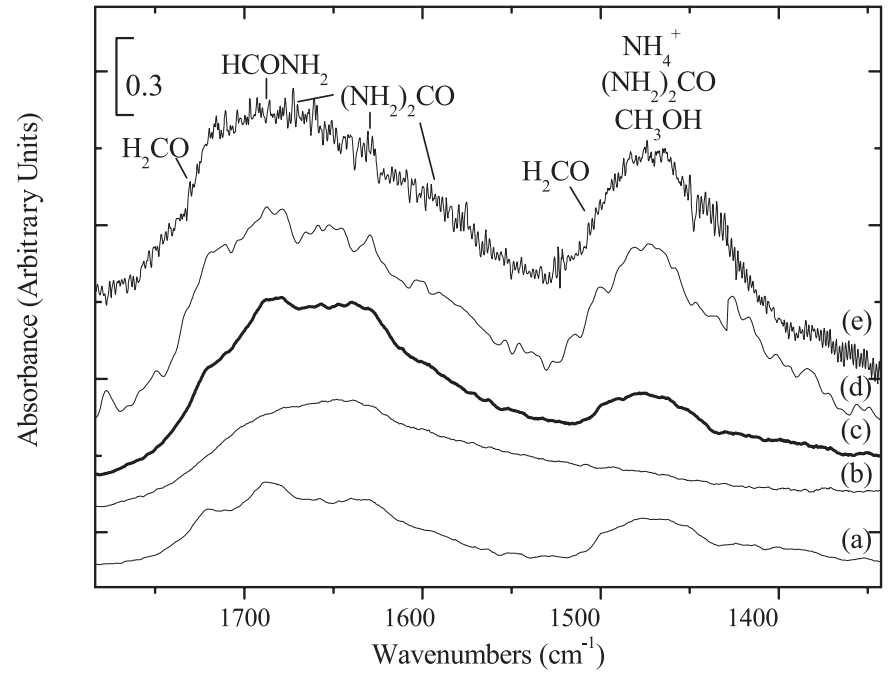

Fig. 4. Comparison between the e) SWS 06, d) SWS 01, ISO-SWS spectra of the protostellar object NGC 7538 IRS9 (Whittet et al. 1996) with the c) sum at $10 \mathrm{~K}$ corresponding to the addition of the spectra of b) solid $\mathrm{NH}_{3} / \mathrm{H}_{2} \mathrm{O}(10 / 100)$ and a) VUV primary photoproducts of HNCO irradiated (the same as Fig. 2a). The b) spectrum of ammonia hydrate $\left(\mathrm{NH}_{3} \cdot \mathrm{H}_{2} \mathrm{O}\right)$ is multiplied by a factor of 20 to get saturation of the $v \mathrm{OH}$ feature near $3200 \mathrm{~cm}^{-1}$ as observed in the ISO spectrum recorded toward the protostellar object NGC 7538 IRS9. The a) spectrum is multiplied by a factor of 90 in order to get features comparable in intensity to those of the ISO spectrum recorded toward the protostellar object NGC 7538 IRS9. ISO Short Wavelength Spectrometer (ISO-SWS) spectra of NGC 7538 IRS9 have been extracted from the ISO database (http://iso www. estec.esa.nl). The SWS01 (low resolution 500) and SWS06 (high resolution 2000) were recorded on February 23th, 1996 and correspond to a total integration time of 1816 and $6894 \mathrm{~s}$ (SWS01 and 06 respectively).

the $1600-1700 \mathrm{~cm}^{-1}$ range (Dartois \& d'Hendecourt 2001). Thus, to be compared with the NGC 7538 IRS9 spectrum in this range, our previous spectrum (Fig. 4a, the same as Fig. 3a) has been added to that corresponding to the $\mathrm{NH}_{3} / \mathrm{H}_{2} \mathrm{O}$ in a ratio $10 / 100$ (Fig. 4b) to give Fig. 4c. The contributions relative to the $\mathrm{C}=\mathrm{O}$ stretch and $\mathrm{NH}$ bend absorptions of these molecules are both in good agreement with the NGC 7538 IRS9 $1700 \mathrm{~cm}^{-1}$ feature. It is clear that the $1470 \mathrm{~cm}^{-1}$ feature recorded in astrophysical ice is not only the result of $\mathrm{NH}_{4}^{+}$absorption but also of other species (Schutte \& Khanna 2003). Thus, formaldehyde and urea must also contribute to the $1470 \mathrm{~cm}^{-1}$ band. The intensity difference observed between the spectra of the irradiated sample and of NGC 7538 IRS9 could be explained from the methanol $\mathrm{CH}$ bend absorption present in the dust grains and not taken into account in our experiments. The methanol may contribute between 30 and $50 \%$ of this absorption (Dartois et al. 1999).

The formation of these carbonyl compounds can be simply explained from the literature. In the VUV region, two HNCO dissociation channels are allowed, leading respectively to $\mathrm{H}+$ NCO (reaction 1) and HN + CO (reaction 2) (Zhang et al. 1995; Brownsword et al. 1997). Reaction 1 is a source of $\mathrm{H}$ atoms which can play a role in the production of new species. Thus $\mathrm{NH}_{3}$, necessary to form $\mathrm{NH}_{4}^{+} \mathrm{OCN}^{-}$, should be the result of a reaction between $\mathrm{H}$ atoms and successively $\mathrm{NH}$ (induced from reaction 2) and $\mathrm{NH}_{2}$ radicals (Lundell et al. 1998). The HCO radical formation observed during the VUV irradiation process (band at $1850 \mathrm{~cm}^{-1}$ ) (Fig. 2a) (Allamandola et al. 1988) suggests that formaldehyde is produced according to the successive hydrogenation reactions $\mathrm{CO}+\mathrm{H}^{\circ} \rightarrow \mathrm{HCO}^{\circ}$ and $\mathrm{HCO}^{\circ}+\mathrm{H}^{\circ}$ $\rightarrow \mathrm{H}_{2} \mathrm{CO}$. Formamide formation can be explained by the following reactions $\mathrm{H}+\mathrm{HNCO} \rightarrow \mathrm{NH}_{2} \mathrm{CO}$ and $\mathrm{H}+\mathrm{NH}_{2} \mathrm{CO} \rightarrow$ $\mathrm{HCONH}_{2}$ involving the $\mathrm{NH}_{2} \mathrm{CO}$ radical as a transient species. This radical has been detected in matrix isolation experiments (Pettersson et al. 1999). Finally, we assume that urea formation can result from a recombination between $\mathrm{NH}_{2} \mathrm{CO}$ and $\mathrm{NH}_{2}$ radicals formed in the prior reactions. Formamide and urea formation require the intervention of at least three and four molecules of HNCO in the mechanism.

\section{Conclusions}

Contribution of formamide and urea can be directly detected at low temperatures in the cold dust grains in NGC 7538 IRS9 especially from their strongest bands located at 1700, 1675, 1630 and $1590 \mathrm{~cm}^{-1}$. These values can be used to find traces of these molecules inside other astrophysical ices. The detection of urea in our work corroborates the results obtained earlier by Agarwal et al. (1985) who identified urea by GC-MS analysis of the products obtained from the photolysis at $10 \mathrm{~K}$ of $\mathrm{CO} / \mathrm{NH}_{3}$ mixtures.

The non-detection of HNCO in interstellar grains may be due to its high reactivity with $\mathrm{NH}_{3}$ at temperatures as low as $10 \mathrm{~K}$ to form $\mathrm{NH}_{4}^{+} \mathrm{OCN}^{-}$and its dissociation under VUV irradiation to form the products mentioned.

The results of these experiments have important implications for both interstellar and cometary chemistry. The organic molecules detected here, mainly formamide and urea, are biologically interesting hand may help establish the importance of extraterrestrial sources of organic material for the Earth prior to the onset of life. Urea plays a large part in the prebiotic synthesis of pyrimidines (Robertson \& Miller 1995) which have been detected, for example, in the Murchison meteorite (Stock \& Schwartz 1981).

Acknowledgements. The authors would like to thank the PCMI National Program (Programme National de Physique et Chimie du Milieu Interstellaire) for their financial support.

\section{References}

Agarwal, V. K., Schutte, W. A., Greenberg, J. M., et al. 1985, Origin of life, 16, 21

Allamandola, L. J., Sandford, S. A., \& Valero, G. J. 1988, Icarus, 76, 225

Bernstein, M. P., Sandford, S. A., \& Allamandola, L. J. 2000, ApJ, 542,894

Brownsword, R. A., Hillenkamp, M., Laurent, T., Vatsa, R. K., \& Volpp, H. R. 1997, J. Chem. Phys., 106, 4436

Bukowska, J. 1979, Spectrochim. Acta, 35A, 985

Cottin, H., Moore, M. H., \& Benilan, Y. 2003, ApJ, 590, 874

Dartois, E., Schutte, W. A., Geballe, T. R., et al. 1999, A\&A, 342, L32

Dartois, E., \& d'Hendecourt, L. 2001, A\&A, 365, 144

d'Hendecourt, L., Allamandola, L. J., Greenberg, J. M., \& Grim, R. J. A. 1986, A\&A, 158, 119 
d'Hendecourt, L., \& Dartois, E. 2001, Spectrochim. Acta, 57A, 669

Gibb, E. L., Whittet, D. C. B., Schutte, W. A., et al. 2000, ApJ, 536, 347

Greenberg, J. M. 2002, Surf. Sci., 500, 793

Grim, R. J. A., Greenberg, J. M., de Groot, M. S., et al. 1989, A\&A, 78,161

Hagen, W., Allamandola, L. J., \& Greenberg, J. M. 1979, Ap\&SS, 65, 215

Hudson, R. L., Moore, M. H., \& Gerakines, P. A. 2001, A\&A, 550, 1140

Jacox, M. E., \& Milligan, D. E. 1964, J. Chem. Phys., 40, 2457

Jenniskens, P., Baratta, G. A., Kouchi, A., et al. 1993, A\&A, 273, 583

Keane, J. V., Tielens, A. G. G. M., Boogert, A. C. A., Schutte, W. A., \& Whittet, D. C. B. 2001, A\&A, 376, 254

Lowenthal, M. S., Khanna, R. K., \& Moore, M. H. 2002, Spectrochim. Acta Part A, 58, 73

Lundell, J., Krajewska, M., \& Räsänen, M. 1998, J. Phys. Chem. A, 102,6643
Novozamsky, J. H., Schutte, W. A., \& Keane, J. V. 2001, A\&A, 379, 588

Pettersson, M., Khriachtchev, L., Jolkkonen, S., \& Räsänen, M. 1999, J. Phys. Chem. A, 103, 9154

Raunier, S., Chiavassa, T., Marinelli, F., Allouche, A., \& Aycard, J. P. 2003, Chem. Phys. Lett., 368, 594

Robertson, M. P., \& Miller, S. L. 1995, Nature, 375, 772

Rousseau, B., van Alsenoy, C., Keuleers, R., \& Desseyn, H. O. 1998, J. Phys. Chem. A, 102, 6540

Schutte, W. A., \& Khanna, R. K. 2003, A\&A, 398, 1049

Soifer, B. T., Puetter, R. C., Russel, R. W., et al. 1979, ApJ, 232, L53

Stoks, P. G., \& Schwartz, A. W. 1981, Geochim. Cosmochim. Acta, 45,563

Whittet, D. C. B., Schutte, W. A., Tielens, A. G. G. M., et al. 1996, A\&A, 315, L357

Winnewisser, G., \& Kramer, C. 1999, Space Sci. Rev., 90, 181

Zhang, J., Dulligan, M., \& Wittig, C. J. 1995, J. Phys. Chem., 99, 7446 